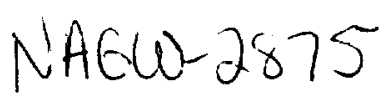

\title{
Dependence of the Normalized Radar Cross Section of Water Waves on Bragg Wavelength-Wind Speed Sensitivity

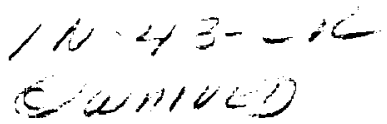

David G. Long, Member, IEEE, R. Scott Collyer, Ryan Reed, Member, IEEE, and David V. Arnold

\begin{abstract}
Measurements of the normalized radar cross section $\left(\sigma^{\circ}\right)$ made by the YSCAT ultrawideband scatterometer during an extended deployment on the Canada Centre for Inland Waters (CCIW) Research Tower located at Lake Ontario are analyzed and compared with anemometer wind measurements to study the sensitivity of $\sigma^{\circ}$ to the wind speed as a function of the Bragg wavelength. This paper concentrates on upwind and downwind aximuth angles in the wind speed range of $4.5-12 \mathrm{~m} / \mathrm{s}$. While YSCAT collected measurements of $\sigma^{\circ}$ at a variety of frequencies and incidence angles, this paper focuses on frequencies of 2.0, 3.05, 5.30, 10.02, and $14.0 \mathrm{GHz}$ and incidence angles within the Bragg regime, $30-50^{\circ}$. Adopting a power law model to describe the relationship between $\sigma^{\circ}$ and wind speed, both wind speed exponents and upwind/downwind $(u / d)$ ratios of $\sigma^{\circ}$ are found using least squares linear regression. The analysis of the wind speed exponents and $u / d$ ratios show that shorter Bragg wavelengths $(\Lambda<4 \mathrm{~cm})$ are the most sensitive to wind speed and direction. Additionally, vertical polarization (V-pol) $\sigma^{\circ}$ is shown to be ore sensitive to wind speed than horizontal polarization (Hpol) $\sigma$, while the $H$-pol $u / d$ ratio is larger than the $V$-pol $u / d$ ratio.
\end{abstract}

\section{INTRODUCTION}

QCATTEROMETERS are active microwave radars that transmit a radar signal toward a target and then measure the energy reflected or scattered back to the sensor. The scattered power measurements are converted into a parameter called the normalized radar cross section $\left(\sigma^{\circ}\right) . \sigma^{\circ}$ is a function of the surface roughness and electrical properties. For the water surface, the roughness is a function of wind speed and direction but is influenced by slicks and other environmental parameters. Given scattered power measurements at several azimuth angles, it is possible to infer the corresponding wind vector [13]. Geophysical model functions attempt to describe the relationship between wind vectors and backscatter. A variety of model functions have been proposed. Some are based solely on empirical data, while others use a combination of theory and empiricism. However, none of the model functions have been able to completely describe the relationship between wind vectors and backscatter.

Deficiencies in the understanding of complex air-sea interactions which affect microwave backscatter from the ocean surface have led to numerous experiments to understand the

Manuscript received January 10, 1994. This work was supported by the NASA Innovative Research Program under Contract NAGW-2875.

The authors are with the Electrical and Computer Engineering Department. Brigham Young University, Provo, UT 84602-4099 USA.

Publisher Item Identifier S 0196-2892(96)02851-3. geophysical model function. Experiments have been conducted using scatterometer data obtained from airplane circle flights, ocean platforms, and water wave tank experiments. Most scatterometers have operated at $\mathrm{Ku}$-band (14 Ghz), C-band (5 Ghz), or X-band (10 Ghz) microwave frequencies [1], [6], [8]-[10]. Some experiments have also been conducted at $\mathrm{L}$ band (1-2 GHz) and Ka-band (35 GHz) [11], [12]. However, all of these frequencies have not been used together in a single experiment.

We have developed an ultrawide band scatterometer, called YSCAT, which can be operated at any frequency from 2-18 $\mathrm{GHz}$, making measurements at a variety of incidence and azimuth angles. This system was recently deployed for 6 months on the Canada Center for Inland Waters (CCIW) Research Tower at Lake Ontario [3], [5]. The multifrequency capability of YSCAT provides an excellent opportunity to study the dependence of $\sigma^{\circ}$ on frequency at different incidence angles. In addition, accurate in-situ weather data are available. The recent YSCAT deployment has provided a large data set for model function studies. This paper presents results on the wind speed sensitivity of $\sigma^{\circ}$ from approximately three months of YSCAT data.

The paper is organized as follows. Section II provides a brief background. Section III then discusses the YSCAT instrument. Section IV discusses the data analysis approach while Section V summarizes the results. Conclusions are provided in Section VI.

\section{BACKGROUND}

Scatterometers transmit electromagnetic energy and measure the amount of power scattered off of a surface. The reflected power is related to $\sigma^{\circ}$ using the radar equation [17]. For moderate incidence angles $\left(20-60^{\circ}\right)$ at microwave frequencies, the sea surface scattering is primarily dependent on small scale (1-15 cm) gravity/capillary waves due to Bragg scattering. In this paper we will concentrate on moderate incidence angles.

In Bragg scattering theory, the backscatter return is assumed to be caused from the water wave component which is in resonance with the incident radiation. In first order Bragg theory, the resonant water wavelength $\Lambda$ is related to electromagnetic wavelength $\lambda$ by

$$
\Lambda=\frac{\lambda}{2 \sin (\theta)}
$$


TABLE I

Bragg WaVElength IN Centometers as a Function of Frequency and InCIDENCE ANGLE

\begin{tabular}{|c|c|c|c|}
\hline $\begin{array}{c}\text { Frequency } \\
(\mathrm{GHz})\end{array}$ & \multicolumn{4}{|c|}{ Incidence Angle } \\
$30^{\circ}$ & $40^{\circ}$ & $50^{\circ}$ \\
\hline 2.00 & 15.00 & 11.67 & 9.79 \\
\hline 3.05 & 9.84 & 7.65 & 6.42 \\
\hline 5.30 & 5.66 & 4.40 & 3.69 \\
\hline 10.02 & 3.00 & 2.33 & 1.96 \\
\hline 14.00 & 2.14 & 1.67 & 1.40 \\
\hline
\end{tabular}

where $\theta$ is the incidence angle. For microwave frequencies of 2-18 GHz, and moderate incidence angles, the Bragg wavelength varies from approximately $1-20 \mathrm{~cm}$, a range which includes capillary and short gravity waves. Table I shows the water Bragg wavelengths measured in this study. As discussed later, the Bragg wavelength can be a useful compact parameter to examine the backscatter versus radar parameters; however, it must be noted that while Bragg scattering may be the dominate scattering mechanism, other scattering mechanisms and hydrodynamic modulation also affect the radar backscatter.

In wind scatterometry geophysical model functions are used to relate $\sigma^{\circ}$ to the surface wind vector. In general, $\sigma^{\circ}$ is a function of polarization $(p), \mathrm{EM}$ frequency $(f)$, incidence angle $(\theta)$, relative azimuth angle $(\chi)$, wind speed $(U)$, and possibly other parameters $(\cdots)$, i.e.,

$$
\sigma^{o}=\mathcal{F}(f, \theta, \chi, p, \cdots) \text {. }
$$

The precise form for $\mathcal{F}$ is still debated. However, most existing model functions have some generalized power-law dependence. A strict power-law formulation may not hold at all wind speeds, as $\sigma^{\circ}$ may fall off rapidly or low wind speeds and saturate or decrease at high wind speeds [4]. However, for midrange wind speeds $(5-16 \mathrm{~m} / \mathrm{s})$ experiments have shown good agreement with the power law model [4], [8]. Since we are interested only in mid-range wind speeds and moderate incidence angles, we have adopted a very simple power-law model function to analyze the wind speed sensitivity of $\sigma^{\circ}$ (see [3], [19])

$$
\sigma^{o}=A(f, \theta, \chi, p) U^{\gamma(f, \theta, \chi, p)} .
$$

In this equation $\gamma$ is referred as to as the wind exponent where the $\gamma$ function arguments $(f, \theta, \chi, p)$ emphasize the dependence on the electromagnetic frequency, incidence angle, relative azimuth angle, and polarization. The wind exponent gives a measure of how sensitive $\sigma^{\circ}$ is to wind speed.

We will consider only two wind directions, upwind and downwind. Previous experiments have shown that $\sigma^{o}$ varies with relative azimuth as approximately $\cos (2 \chi)$ with a small asymmetry between the value of $\sigma^{\circ}$ at upwind $\left(\chi=0^{\circ}\right)$ and downwind $\left(\chi=180^{\circ}\right)$ with $\sigma^{\circ}$ at upwind being lightly larger than $\sigma^{\circ}$ at downwind. The difference between $\sigma^{\circ}$ at upwind and downwind (upwind/downwind ratio) is used to remove the $180^{\circ}$ directional ambiguity from the wind direction inherent in the wind retrieval problem [13]. Thus, the upwind/downwind ratio is an important parameter which will be investigated.

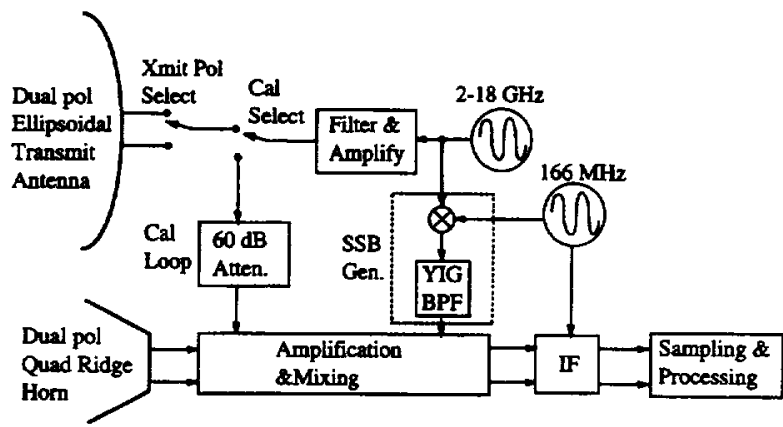

Fig. 1. YSCAT functional block diagram.

\section{YSCAT SYSTEM AND EXPERIMENT DESCRIPTION}

In this section, we describe the YSCAT instrument, its measurement scheme, the environmental sensors, and the experiment site. The next section describes the data analysis.

YSCAT is an ultrawideband CW-Doppler radar scatterometer developed at Brigham Young University that can be operated at any frequency from $2-18 \mathrm{GHz}$. It transmits either a $\mathrm{V}$ or $\mathrm{H}$ polarization signal, with a maximum output power of $23 \mathrm{dBm}$. The transmit antenna is a specially designed $3 \mathrm{ft}(0.91$ m) ellipsoidal figure antenna which provides a nearly constant beamwidth of $5^{\circ}$ from $4-18 \mathrm{GHz}$. At $2 \mathrm{GHz}$ the beamwidth approaches $8^{\circ}$. The receive antenna is a conventional quadridge hom with a beamwidth which varies from $45^{\circ}$ to $7.5^{\circ}$ over the $2-18 \mathrm{GHz}$ frequency range. A function block diagram of YSCAT is given in Fig. 1.

The dual-pol receiver simultaneously receives $\mathrm{V}$-pol and $\mathrm{H}$ pol channels. After amplification and mixing down to a 166 $\mathrm{MHz}$ IF, the signal is split into in-phase and quadrature (I/Q) components which are then mixed down to baseband and low pass filtered at $900 \mathrm{~Hz}$. The filtered baseband V/Q signals are sampled at $2 \mathrm{kHz}$ using 12-b A/D's.

Nominal measurements are one minute long; however, 20 min measurements were also collected. To conserve on disk storage space, the raw data is processed into $10 \mathrm{~Hz}$, or 0.1 $\mathrm{s}$, measurement records consisting of a power measurement in $\mathrm{dB}$, the Doppler centroid, and the Doppler bandwidth in $\mathrm{Hz}$. The power is caiculated by first finding the average of the squares of the individual voltage measurements, and then converting the value to decibels. The Doppler centroids and bandwidths are found using first and second moment estimation techniques (see [7]).

YSCAT was mounted $6.26 \mathrm{~m}$ above the water surface. The incidence and azimuth angles of the antennas are controlled using stepping motors. The incidence angle can be adjusted from nadir $\left(0^{\circ}\right)$ to greater than $90^{\circ}$. The azimuth angle can be set to $\pm 80^{\circ}$ from looking straight out from the platform.

Data is also collected from an array of environmental sensors. These include two separate anemometers, an aspirated temperature sensor, a humidity gauge, a water temperature gauge, and a rain gauge. The data from these sensors are collected and averaged into $30 \mathrm{~s}$ measurements which are then recorded in the radar files. The anemometers, which provide the $U_{10}$ wind speeds used in this paper, are mounted at a height of approximately $10 \mathrm{~m}$ above the water surface. 


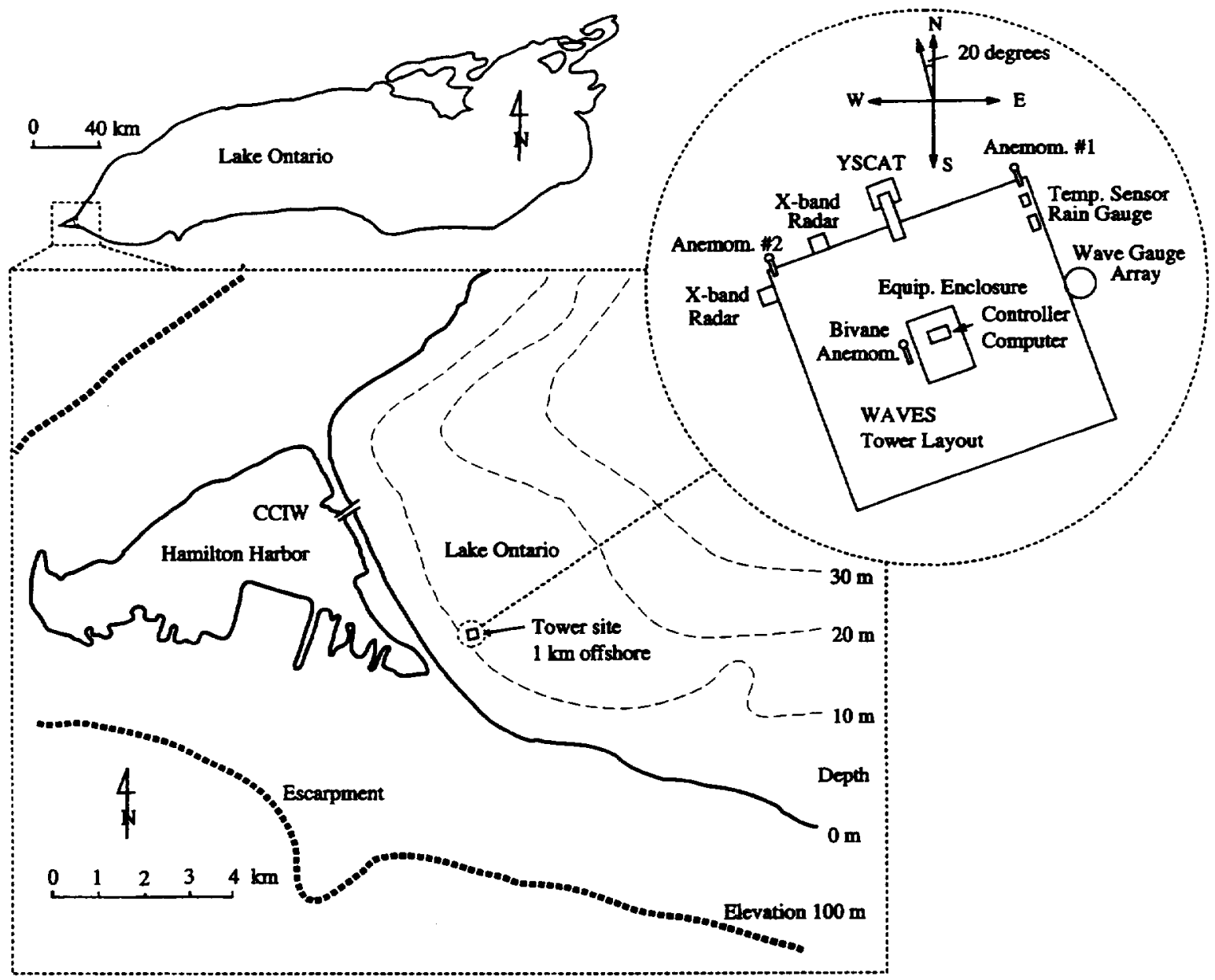

Fig. 2. Location and layout of the WAVES tower on Lake Ontario with the mounting location of YSCAT and other environmental sensors shown. The two X-band radars are part of a separate experiment.

YSCAT was deployed from May 1994 through November 1994 on the CCIW Research Tower located on Lake Ontario (see Fig. 2). The CCIW tower is designed to have minimal impact on the winds and waves [5]. The location provides both long and short fetch wind directions, where the fetch varies from $1-100 \mathrm{~km}$. The long fetch directions are from $50-90^{\circ} \mathrm{T}$. The water depth at the tower location is approximately $12 \mathrm{~m}$.

Because Lake Ontario is a fresh water lake and the tower is located near the shore the response is somewhat different than the open ocean. During the experiment period most significant winds came from short fetch directions $(1-10 \mathrm{~km})$. As a result, a significant long wave field was rarely present. For example the significant wave height was typically less than $0.5 \mathrm{~m}$, while the maximum significant wave height encountered was $2 \mathrm{~m}$; however, this only occurred during a two day period. Since in the open ocean $2 \mathrm{~m}$ wave heights are very common, $\sigma^{\circ}$ measurements from Lake Ontario will be somewhat different than those on the open ocean with respect to the long wave field.

Colton et al. [3] has shown that Lake Ontario waves are typically shorter and steeper than in the open ocean resulting in a higher drag coefficient. This suggests that for a given wind speed the wind stress over the lake will be higher than that of the ocean, resulting in a stronger wind speed sensitivity of $\sigma^{\circ}$. The results of this experiment must be interpreted accordingly.

\section{DATA ANALYSIS}

Approximately three months of data, collected from May 6-August 1 , are analyzed in this paper. During this time inter$\mathrm{val}$, the significant wave height was generally small, typically less than $0.5 \mathrm{~m}$ with the exception of a two day period. The wind speed ranged up to $14 \mathrm{~m} / \mathrm{s}$ from virtually all directions. The air temperature varied from $10-30^{\circ} \mathrm{C}$ and the water temperature changed from $9^{\circ} \mathrm{C}$ to a high of approximately $20^{\circ} \mathrm{C}$.

Previous research has suggested that $\sigma^{\circ}$ may be affected by the air-sea temperature difference [8], [9], the water temperature [4], or the long wave field [10]. However, the importance of these effects is still a matter of debate. During the three month data set the air-sea temperature difference was always positive or neutral and the long wave field was usually small. As a result, the current data set does not allow an adequate comparison between the different cases of air-sea temperature difference and long wave field and these are ignored in the data analysis. 

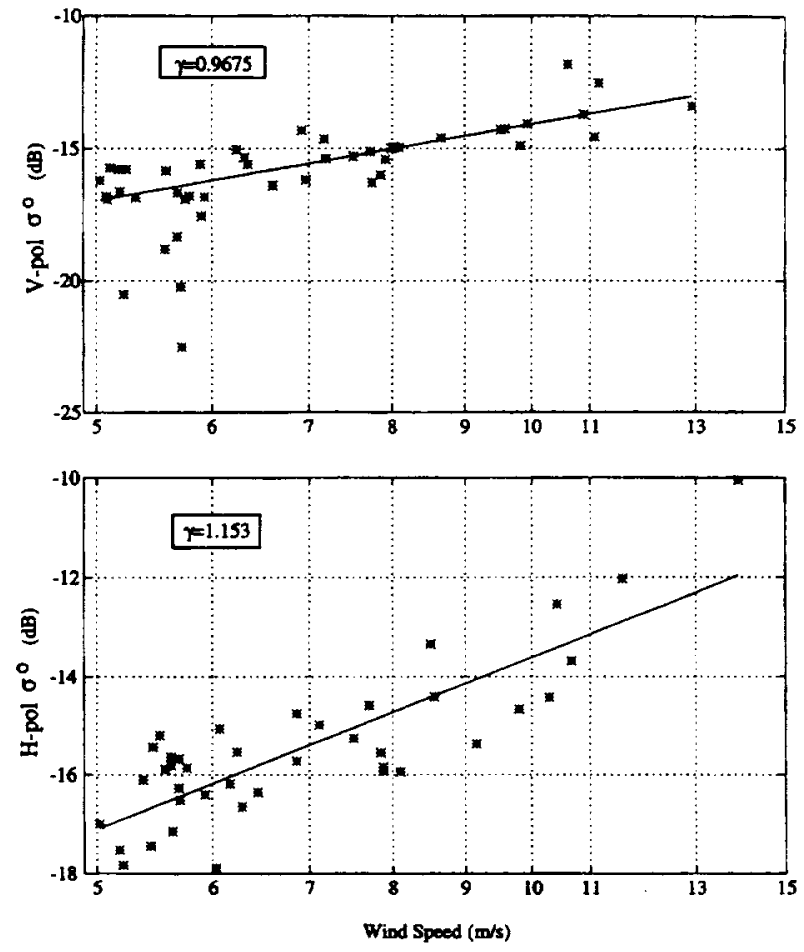

Fig. 3. Downwind measurements of $\sigma^{\circ}$ at $3.05 \mathrm{GHz}$ and $30^{\circ}$ incidence versus wind speed. The line is a linear regression of the points using a $\pm 2 \sigma$ outlier correction (see text). Corrections for the absolute calibration of the scatterometer have not been included in the $\sigma^{\circ}$ values shown.

Rain can effect the value of $\sigma^{\circ}$ by attenuating the radar signal and by effecting the sea surface. In addition, the radar gain can be effected by the presence of water on the antenna reflector. Thus, for this study, measurements contaminated by rain are discarded in a manual editing process [2]. To investigate the wind speed sensitivity of $\sigma^{\circ}$, only $\chi$ values of $\pm 10^{\circ}$ of upwind and downwind with wind speeds greater than $4.5 \mathrm{~m} / \mathrm{s}$ are used.

Since the focus of this paper is on wind speed sensitivity and the issues associated with the absolute calibration of a scatterometer too involved to incorporate into this paper, the analysis techniques we have used have been chosen to avoid dependence on the absolute calibration of the scatterometer. The $\sigma^{\circ}$ values presented in this paper have not been corrected for the absolute calibration of the scatterometer.

\section{A. Regression Analysis}

To investigate the sensitivity of $\sigma^{\circ}$ at different frequencies, the measurements in each record are first averaged to produced one minute averages. All averaging is done in linear space since averaging in log space may produce too low a value, resulting in errors up to 3 or $4 \mathrm{~dB}$ [8]. Plots of $\sigma^{\circ}$ (in decibels) versus the $\log$ of wind speed display a linear trend. Fig. 3 gives an example of $\mathrm{V}$-pol and $\mathrm{H}$-pol data at downwind, 3.05 $\mathrm{GHz}$, and $30^{\circ}$ incidence. Wind speed is the wind at $10 \mathrm{~m}$, $U_{10} . \sigma^{\circ}$ values shown in this plot have not been corrected for the absolute calibration of the $\mathrm{V}$ and $\mathrm{H}$ pol channels of the scatterometer.
To compute the slope $\gamma$ of the linear fit, the simple regression model

$$
\sigma_{i}^{o}=A_{0}+\gamma 10 \log _{10}\left(U_{i}\right)+e_{i}
$$

is used, where $\sigma_{i}^{\circ}$ is expressed in decibels, $A_{0}$ is a constant, $\gamma$ is the slope, $U$ is the wind speed in $\mathrm{m} / \mathrm{s}$, and $e_{i}$ represents the random error term which is assumed to be normally distributed. This corresponds to linear regression of $\sigma^{\circ}$ in decibels versus wind speed in normal space. In computing the regresssion, outliers are discarded. Outliers are defined as points that are more than $\pm 2 \sigma$ from linear regression line determined in an initial regression fit using all the points [2]. A summary of the regression slopes is given in Fig. 4. Note that the $\gamma$ values are not dependent on the absolute calibration of the scatterometer.

Confidence intervals were computed based on the number of independent samples in each one minute measurement which is determined from the correlation times of the microwave backscatter signal. Assuming that the correlation function falls off as $e^{-a \tau}$, the correlation time is the time required for the correlation function to decay to $1 / e$ of its maximum value. Typically, the correlation time is a function of frequency with shorter correlation times associated with higher frequencies. We compute the correlation times for each study frequency below.

\section{B. Correlation Times}

Plant et al. [14] state that at X-band the correlation time is approximately $10 \mathrm{~ms}$. This suggests that at X-band, the 0.1 $s$ samples of power measurements collected by YSCAT are essentially uncorrelated. Following the methods of Plant $e t$ al., the correlation times can be found from an estimate of the radial velocity spread which is given by

$$
\delta_{v_{r}}=\frac{\lambda\left(\bar{f}_{2}\right)^{1 / 2}}{2}
$$

where $\delta_{v_{r}}$ is the radial velocity spread, $\lambda$ is the microwave wavelength, and $\bar{f}_{2}$ is the second moment of the Doppler spectrum. The value of $\bar{f}_{2}$ can be found from the Doppler bandwidth estimate that is collected along with each power measurement. Finally, the correlation time, $t_{0}$ is found from

$$
t_{0}=\frac{\lambda}{4 \sqrt{\pi} \delta_{v_{r}}}
$$

Using (3) and (4), correlation times are computed with the aid of an extended length collection mode. In this mode approximately $18 \mathrm{~min}$ of consecutive $0.1 \mathrm{~s}$ samples are collected for each frequency and polarization. Data collected in this mode spans incidence angles ranging from $30-50^{\circ}$ and wind speeds from $5-9 \mathrm{~m} / \mathrm{s}$. The resulting average correlation times and standard deviations are shown in Table II. Note that even for the largest correlation time $(13.5 \mathrm{~ms})$ at 3.05 $\mathrm{GHz}, t_{0}+\sigma$ is less than $20 \mathrm{~ms}$. A $20 \mathrm{~ms}$ correlation time implies that one half of the $0.1 \mathrm{~s}$ samples can be considered uncorrelated. Using $20 \mathrm{~ms}$ as a conservative estimate for all frequencies and incidence angles considered, confidence intervals are calculated assuming 300 of the 600 measurements 

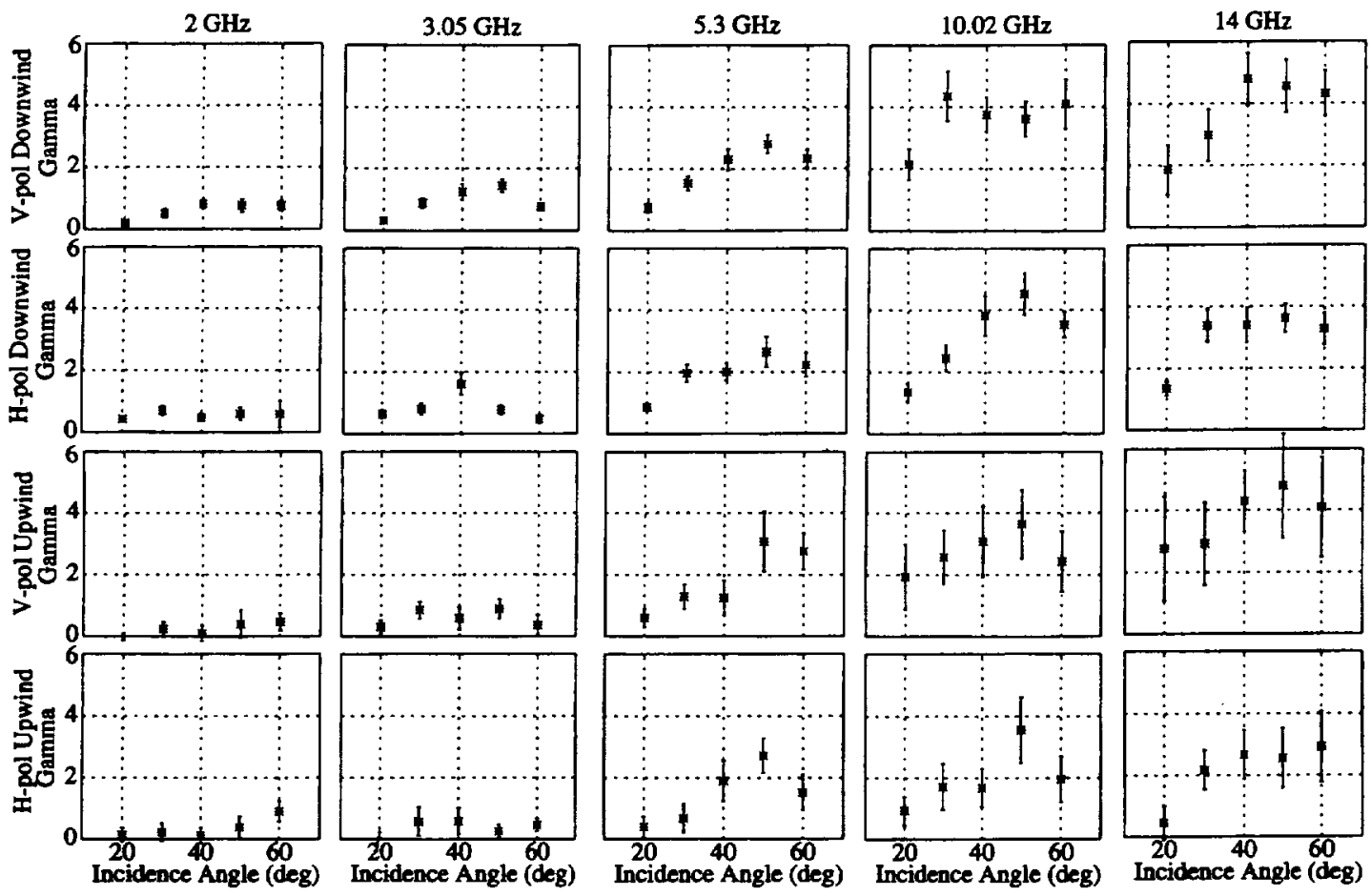

Fig. 4. $\gamma$ versus incidence angle $(\theta)$ for various frequencies, polarizations, and wind directions.

TABLE II

average Correlation Times $\left(t_{0}\right)$ and Standard Deviations $(\sigma) \mathbb{N}$ ms From $0.1 \mathrm{~s}$ POWER MEASUREMENTS FROM 30-50 $0^{\circ}$ INCIDENCE

\begin{tabular}{|c|c|c|c|c|}
\hline Frequency & \multicolumn{2}{|c|}{$\mathrm{V}$ - Pol } & \multicolumn{2}{c|}{$\mathrm{H}$ - Pol } \\
\cline { 2 - 5 }$(\mathrm{GHz})$ & $t_{0}$ & $\sigma$ & $t_{0}$ & $\sigma$ \\
\hline 2.00 & 11.9 & 5.7 & 12.2 & 5.9 \\
\hline 3.05 & 13.5 & 5.8 & 10.0 & 4.4 \\
\hline 5.30 & 11.6 & 5.8 & 12.0 & 5.4 \\
\hline 10.02 & 7.20 & 3.5 & 11.0 & 5.0 \\
\hline 14.00 & 6.10 & 3.2 & 7.8 & 3.5 \\
\hline
\end{tabular}

made in one minute are independent. Although assuming that uncorrelation implies independence is not completely correct, the resulting error is small [15]. The resulting $95 \%$ confidence interval is typically less than $\pm 0.1 \mathrm{~dB}$ for the $\sigma^{\circ}$ regression. In all cases the $95 \%$ confidence interval is less than $\pm 1 \mathrm{~dB}$.

\section{DATA ANALYSIS RESULTS AND DISCUSSION}

The sensitivity to wind speed is conveniently analyzed through the wind speed exponent $(\gamma)$ computed for both upwind and downwind. The sensitivity to wind direction can be expressed in terms of the wind speed exponent and $A_{0}$. We have analyzed the $\sigma^{\circ}$ measurements to investigate: 1) the wind speed exponent $(\gamma)$ as a function of incidence angle; 2) $\gamma$ as a function of Bragg wavelength; 3) differences between $\gamma$ for $\mathrm{V}$ pol, H-pol, upwind, and downwind; and 4) upwind/downwind $\sigma^{\circ}$ ratio as a function of Bragg wavelength and wind speed.

\section{A. Wind Exponent $(\gamma)$}

The least squares linear fit is found for each frequency, incidence angle and polarization for both upwind and downwind cases. The results are summarized in Fig. 4 . In this figure $\gamma$ values at $20^{\circ}$ and $60^{\circ}$ have been included to give a wider range of incidence angles even though these are outside of the primary incidence angle study range of $30-50^{\circ}$. The error bars represent $90 \%$ confidence limits determined from the standard error of the slopes. We note that the $\mathrm{V}$-pol 10 and $14 \mathrm{GHz}$ measurements exhibit considerable scatter. This phenomena has also been observed by Plant et al. [11] who note that the data spread at X-band is always considerable when wind speed is used as the independent variable. Since there is much less scatter in the data at lower frequencies, it is suggested that $\sigma^{\circ}$ at $\mathrm{X}$ and $\mathrm{Ku}$ bands is more sensitive to other unmodeled geophysical parameters than it is at lower frequencies.

\section{B. Effect of Incidence Angle on $\gamma$}

Previous studies have suggested that $\gamma$ increases with increasing incidence angle. Unal et al. [18] found that $\gamma$ increases with incidence angle for $\mathrm{H}$-pol, but that the trend is not as obvious at V-pol. Chaudhry and Moore [1] (hereafter $\mathrm{CM}$ ) concluded that $\gamma$ increased rapidly at lower incidence angles, but at larger angles $\left(\theta>30^{\circ}\right) \gamma$ varied little. YSCAT data (Fig. 4) show similar results over a broader frequency and incidence angle range for both upwind and downwind directions.

In general, the data show that $\gamma$ increases as $\theta$ goes from $20-50^{\circ}$ and that the slopes are a function of frequency. With the exceptions of $2 \mathrm{GHz}, \mathrm{V}$-pol downwind at $10 \mathrm{GHz}$, and $\mathrm{H}$ pol upwind at $14 \mathrm{GHz}$, the results suggest that $\gamma$ peaks at $50^{\circ}$ and then begins to decrease. This trend is more pronounced at higher frequencies. The results at $3 \mathrm{GHz}$ suggest the same trend, although it is not as pronounced where $\gamma$ is always 

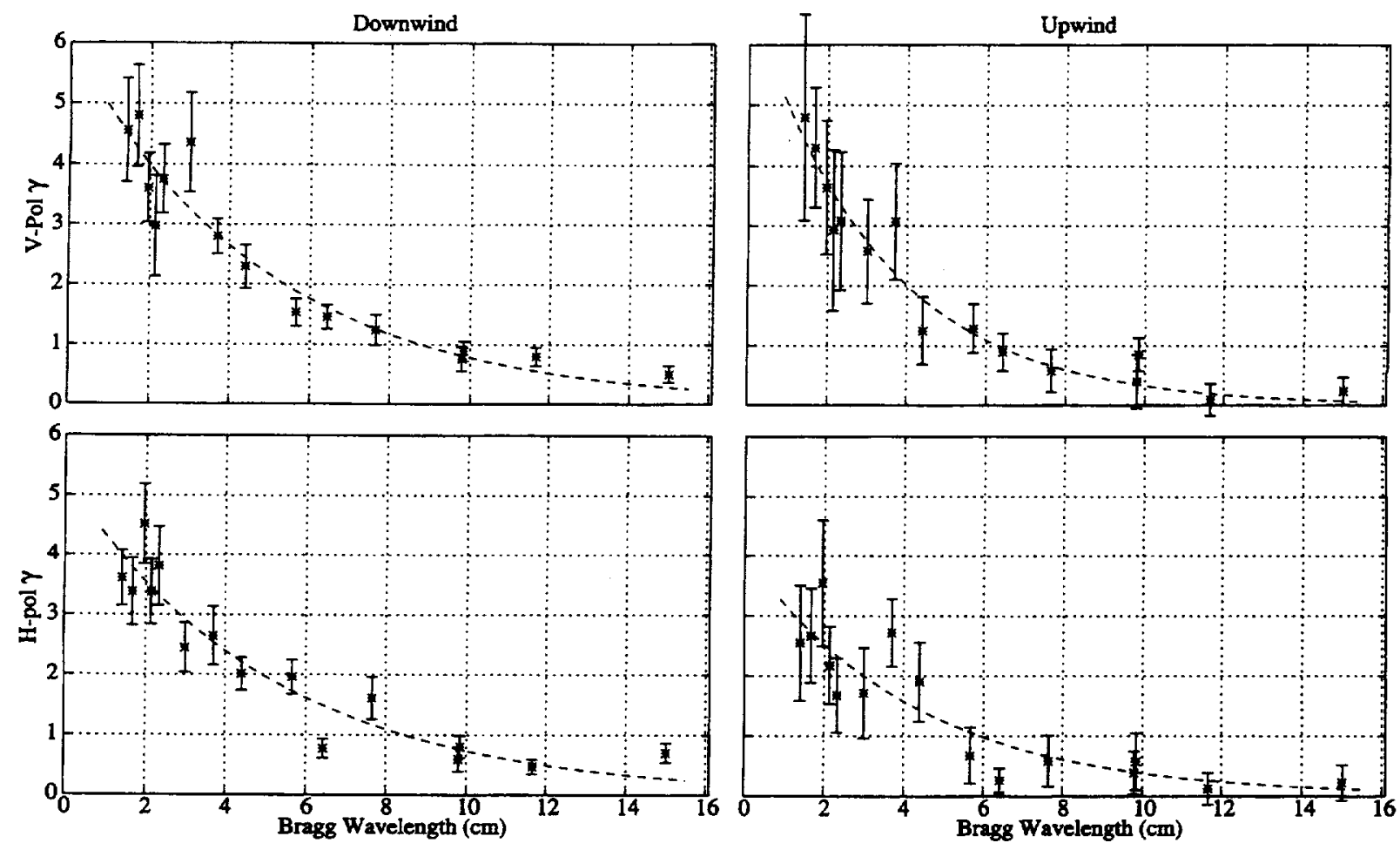

Fig. 5. Wind exponent $(\gamma)$ as function of Bragg wavelength. Only $\gamma$ values from $30-50^{\circ}$ incidence angle are shown. The dashed line is the least squares exponential fit to the data.

less than one. The increase with incidence angle of the V-pol values is consistent with the results found by [18]; however, an increasing trend is also apparent at $\mathrm{H}$-pol. This differs from Unal et al. [18] who state that V-pol did not have as large an increase as $\mathrm{H}$-pol. The reasons for this difference are not apparent.

\section{Wind Speed Exponent Versus Bragg Wavelength}

The previous section shows that $\gamma$ is a function of incidence angle, azimuth angle, polarization and frequency. For moderate incidence angles $\left(30-50^{\circ}\right)$ the surface scatter is dominated by Bragg scattering. With this in mind, the behavior of $\gamma$ can be examined with respect to the Bragg wavelength. The Bragg wavelength provides a compact parameter to combine frequency and incidence angle. Our use of the Bragg wavelength simplifies presentation of the data. We note, however, that other scattering mechanisms (e.g., wedge scattering and wave breaking) and hydrodynamic modulation of the small wave field by long waves can also effect the observed $\sigma^{\circ}$. Parameterizing the observed backscatter by Bragg wavelength neglects these effects. Nevertheless, doing so can provide insights into the wind-speed behavior of the backscatter as a function of the radar parameters included in the Bragg wavelength parameter as well the surface wave spectrum. We also note that the derived wind exponents do not represent a physical property of the surface but rather a particular parameterization of the cross-section of the surface.

Using only incidence angles from $30-50^{\circ}$, the values of $\gamma$ are plotted against Bragg wavelength in Fig. 5. Also shown on each plot is the least squares exponential fit to the data. The error bars display the $90 \%$ confidence levels of the $\gamma$ estimates. The error bars are largest for the upwind cases as a result of the regression being computed with fewer data points compared to the downwind cases. This figure suggests that $\sigma^{\circ}$ is much more sensitive (i.e., $\gamma$ is larger) to wind speed at smaller Bragg wavelengths $(\Lambda<4 \mathrm{~cm})$ than at longer wavelengths. In fact for $\Lambda>8 \mathrm{~cm}, \gamma$ is less than one in all cases. The differences of $\gamma$ between the V-pol, H-pol, upwind, and downwind cases are discussed later.

Surface ocean waves receive energy from the wind, either directly or indirectly through nonlinear interactions with smaller waves. Smaller waves are generally in equilibrium with the wind and will respond more strongly to changes in the wind than will larger waves. Higher wind exponents are indicative of this fact. The Bragg wavelengths of water waves which are very sensitive to changes in the wind speed will correspond to high wind exponents. The finding that $\gamma$ is less than one for $\Lambda>8 \mathrm{~cm}$, suggests that these waves may receive their energy more from nonlinear interactions with smaller waves than directly from the wind.

Fig. 6 displays the comparison between wind exponents calculated from YSCAT data to those found with other experiments. Note that only an upwind comparison is shown, as only a few results from other experiments at downwind are available. In the V-pol case, YSCAT values are greater than all others for $\Lambda<4 \mathrm{~cm}$. For $H$-pol the wind exponents are more comparable to the other results. The fact that YSCAT measurements were taken in a fresh water lake rather than in 

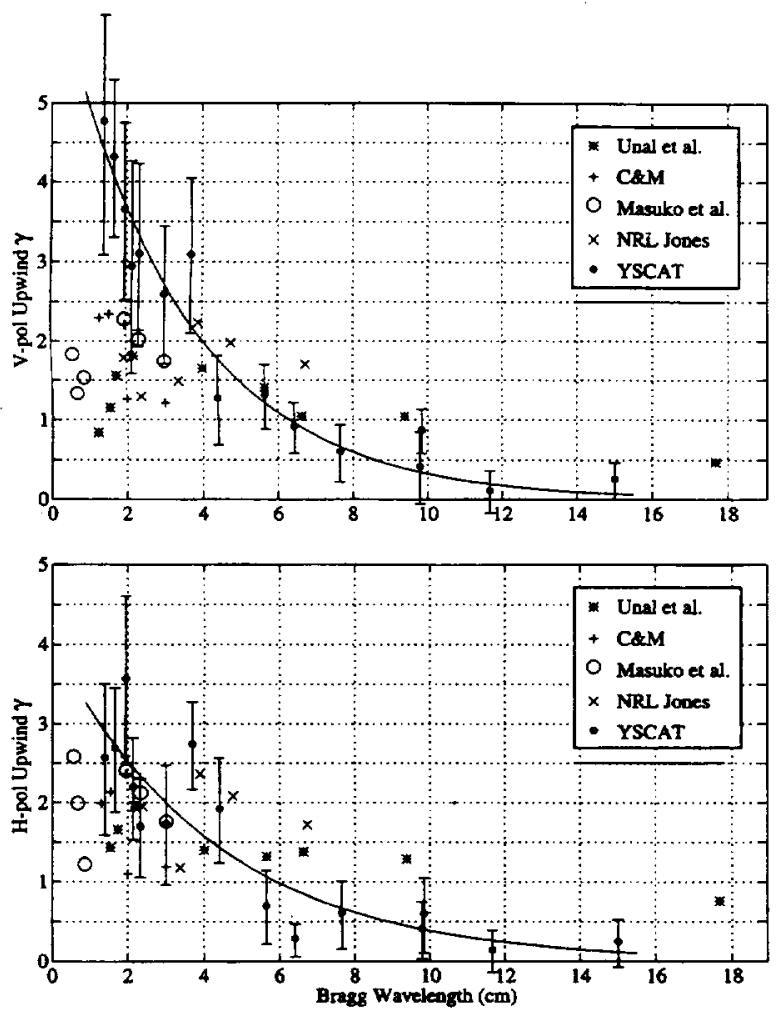

Fig. 6. Comparison of upwind YSCAT wind speed exponents $(\gamma)$ to others for both polarizations.

the open ocean may explain why the exponents are higher. Not only are the water properties different, but in the lake the waves are generally less developed than ocean waves. In a previous experiment on the CCIW Research Tower (WAVES 87) Colton et al. [3] found that $\sigma^{o}$ measured at Lake Ontario had a higher wind speed dependence than those over the open ocean. Analyzing this data, Colton et al. [3] hypothesized that the higher wind speed exponents could be attributed to the difference between the drag coefficient of the open sea and that of Lake Ontario. The drag coefficient on the lake has a higher wind speed dependence because lake waves are often in an active growth stage and are steeper than waves in the ocean. Correcting for the assumed difference in drag coefficient, Colton et al. showed that the wind exponents at $40^{\circ}$ and $60^{\circ}$ incidence angles decreased by almost a factor of 2 . Since wind stress measurements are not currently available, the YSCAT measurements can not be similarly rescaled. However, it is assumed that they would also decrease and be in better agreement with other results.

Both the data from Masuko et al. [12] and Unal et al. [18] suggest that $\gamma$ does not always increase as the Bragg wavelength decreases, but rather decreases as $\Lambda$ becomes less than $2 \mathrm{~cm}$. A drop in $\gamma$ can possibly be explained physically by the fact that for very small waves (purely capillary) the viscous and dissipative effects become more important. As a result the waves are not as sensitive to changes in the wind speed [16]. From the current YSCAT data, it does not appear that $\gamma$ decreases significantly for very short Bragg wavelengths. Further research to resolve this question is needed.
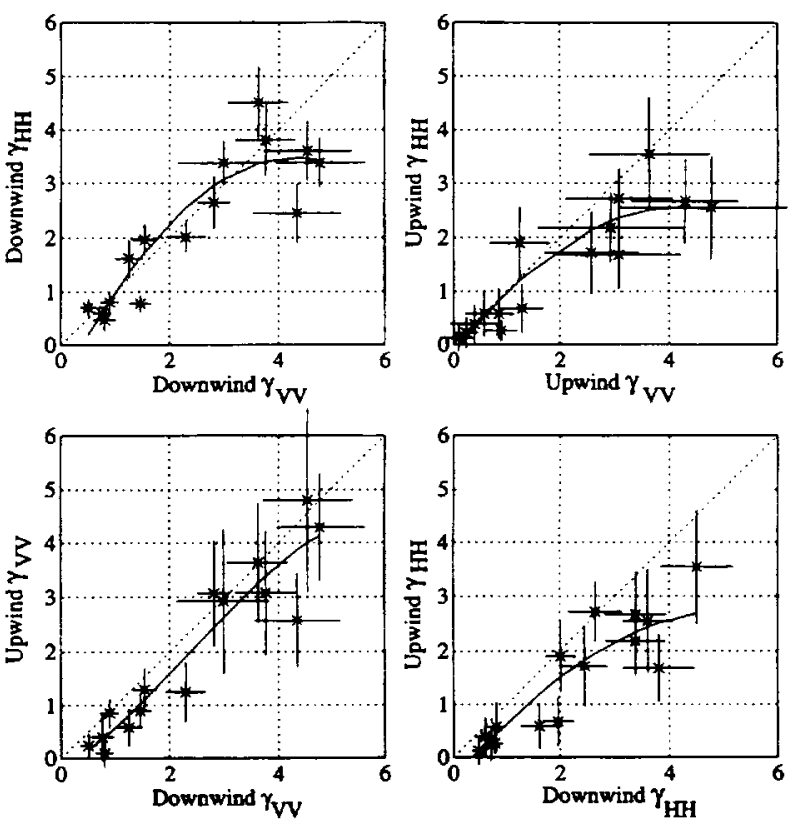

Fig. 7. Comparison of $\gamma_{\mathrm{HH}}$ to $\gamma_{\mathrm{VV}}$ and $\gamma_{L}$ to $\gamma_{D}$ for each polarization.

\section{Differences in $\gamma$ for Upwind, Downwind, and Polarization}

To examine the dependence of $\gamma$ on polarization, the $\mathrm{H}$ pol $\gamma$ (denoted $\gamma_{\mathrm{HH}}$ ) is plotted against the $\mathrm{V}$-pol $\gamma$ (denoted $\gamma_{\mathrm{VV}}$ ) for both upwind and downwind in the upper two plots of Fig. 7. The dotted line represents a perfect agreement between the two polarizations, while the solid line is a least squares second order polynomial fit. For low $\gamma$, the points are close to the $45^{\circ}$ line. However, as $\gamma$ increases, $\gamma_{\mathrm{VV}}$ dominates $\gamma_{\mathrm{HH}}$. This trend is strongest at upwind, and suggests that V-pol is more sensitive to wind speed than $\mathrm{H}$-pol.

The lower two plots of Fig. 7 show the comparison between $\gamma$ at upwind (denoted $\gamma_{U}$ ) and $\gamma$ for downwind (denoted $\gamma_{D}$ ) for both V-pol and H-pol. The V-pol case suggests that $\gamma_{D}$ is slightly larger than $\gamma_{U}$, while at $\mathrm{H}$-pol $\gamma_{D}$ is always larger than $\gamma_{U}$. As will be shown later, the difference between $\gamma_{U}$ and $\gamma_{D}$ has implications regarding the upwind/downwind ratio. In both cases $\gamma_{D}$ is typically larger than $\gamma_{U}$. Since larger $\gamma$ are associated with smaller Bragg wavelengths, this result suggests that for smaller Bragg wavelengths $\mathrm{H}$-pol has a greater change in the upwind/downwind ratio than V-pol as a function of wind speed.

The ratios of $\gamma_{U}$ to $\gamma_{D}$ for both $\mathrm{V}$-pol and $\mathrm{H}$-pol as a function of Bragg wavelength are given in the top plot of Fig. 8 . The dashed and solid lines are least squares fit to the data. Note that in both cases the $\gamma_{U} / \gamma_{D}$ ratio is less than one, but increasing with decreasing Bragg wavelength. This implies that shorter Bragg wavelengths will produce higher upwind/downwind ratios. The lower plot of Fig. 8 illustrates the $\gamma_{\mathrm{HH}} / \gamma_{\mathrm{VV}}$ ratio as a function of Bragg wavelength. An important result is that for small Bragg wavelengths, V-pol is more sensitive to wind speed, particularly for upwind.

Turning to the upwind/downwind $(u / d)$ ratio and using a linear regression fit, the $u / d$ ratio of $\sigma^{\circ}$ can be found for a 

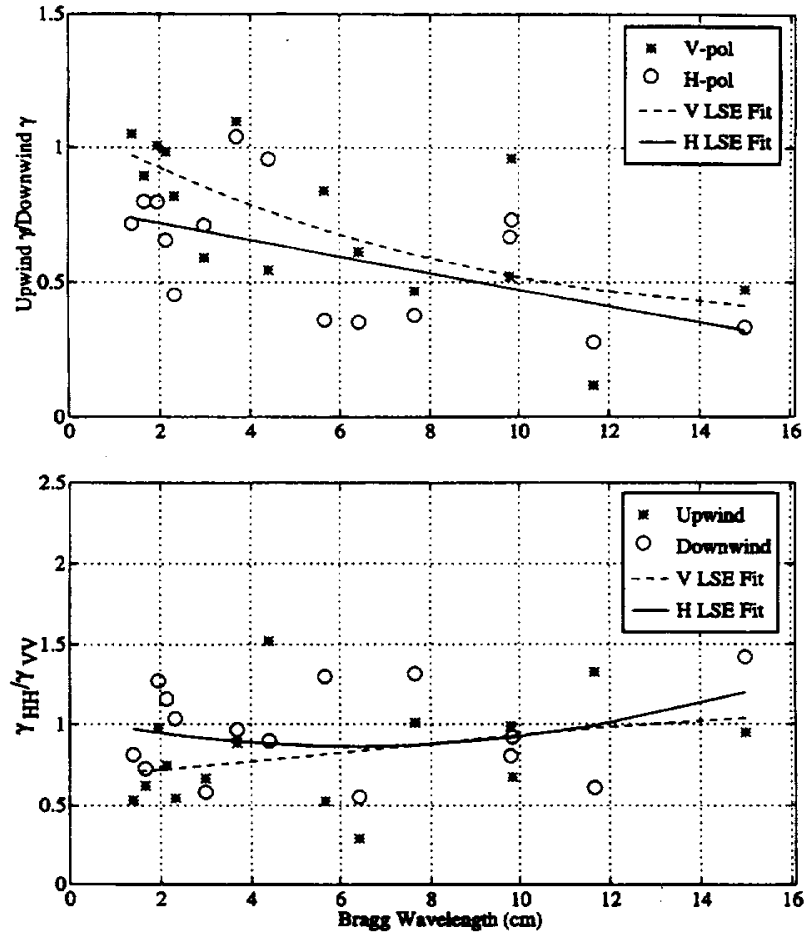

Fig. 8. $\gamma_{\mathrm{HH}} / \gamma_{\mathrm{VV}}$ ratio and $\gamma_{U} / \gamma_{D}$ ratio as function of Bragg wavelength.

given wind speed $(U)$ using

$$
\left(\frac{u}{d}\right)_{\mathrm{dB}}=\left(\sigma_{D}^{o}\right)_{\mathrm{dB}}=A_{U}-A_{D}+\left(\gamma_{U}-\gamma_{D}\right) 10 \log _{10}(U)
$$

where $A_{U}$ and $A_{D}$ are the $A_{0}$ values for the upwind and downwind cases, respectively. Note that the $u / d$ ratio is a function of wind speed and is independent of the absolute calibration of the system. Fig. 9 illustrates the $u / d$ ratio for $U=5 \mathrm{~m} / \mathrm{s}$ and $U=10 \mathrm{~m} / \mathrm{s}$. The solid lines in each plot give the least squares polynomial fit to the data. A first order polynomial is used for the V-pol cases, while a third order polynomial is used for the $\mathrm{H}$-pol cases. The $\mathrm{H}$-pol results show a definite trend of increasing $u / d$ ratio for decreasing Bragg wavelengths. Though the ratio is less than that for H-pol, the $V$-pol $u / d$ ratios generally increase as Bragg wavelengths decrease. In all instances, the $\mathrm{H}$-pol $u / d$ ratios are higher than $\mathrm{V}$-pol. For both the $\mathrm{V}$-pol and $\mathrm{H}$-pol cases, the $u / d$ ratios decrease as the wind speed increases.

Other researchers have found similar results regarding the differences in $\mathrm{H}$-pol and $\mathrm{V}$-pol ratios as well as the increase in $u / d$ with decreasing Bragg wavelength. A brief summary of these results are now presented. Using a C-band V-pol radar, Feindt $e t$ al. [6] found that $u / d$ varies between 0 and 2 $\mathrm{dB}$, but they found no systematic dependence on wind speed. However, using data from Jones and Schroeder [6], Feindt et al. did see a slight increase in $u / d$ with decreasing Bragg wavelength. Masuko et al. [12] at X-band reported that for $V$-pol, $u / d$ varied from $0.42-2.01$ over the incidence angle range of $32-52^{\circ}$. For $\mathrm{H}$-pol over the same incidence range the $u / d$ ratio was approximately $2.5 \mathrm{~dB}$ so their results also show that H-pol $u / d$ is larger than the V-pol case. These reported values of $u / d$ are averaged over a wind speed range of 3-17
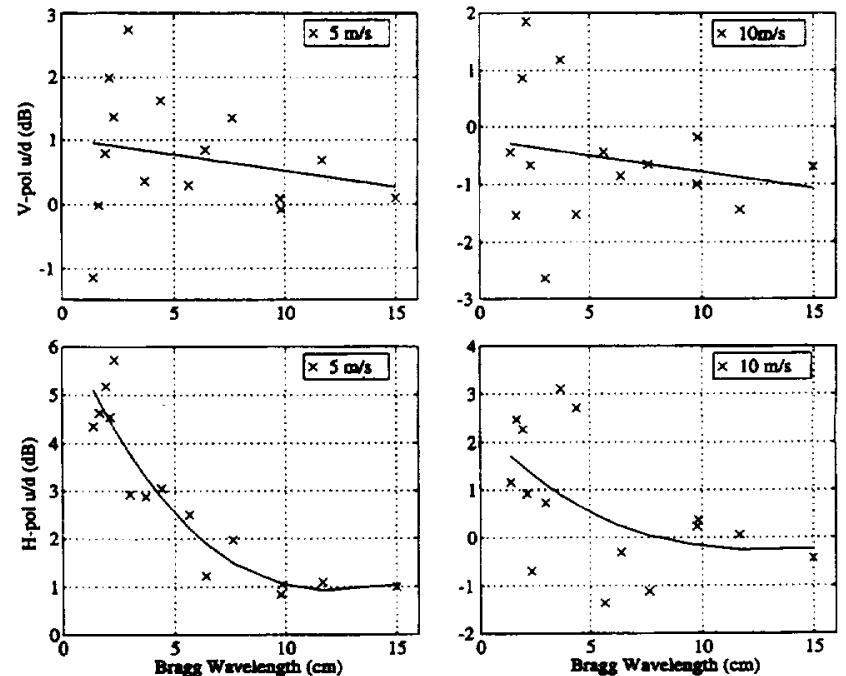

Fig. 9. Upwind/Downwind ratio as function of Bragg wavelength for $U=5$ and $10 \mathrm{~m} / \mathrm{s}$.

$\mathrm{m} / \mathrm{s}$, but they state that above $7-9 \mathrm{~m} / \mathrm{s}$ the $u / d$ ratios appear to decrease with increasing wind speed and become less than unity $(0 \mathrm{~dB})$ for high wind speed cases. Unal et al. [18] report $u / d$ results for both V-pol and H-pol at a wind speed of $10 \mathrm{~m} / \mathrm{s}$ and incidence angles of 30 and $45^{\circ}$. They found that $\mathrm{H}$-pol is larger than $\mathrm{V}$-pol, and that $u / d$ increases with incidence angle, which at a given frequency, is equivalent to decreasing Bragg wavelength. They also report that the difference between $\mathrm{H}$-pol and V-pol $u / d$ decreases with increasing wind speed.

A difference between YSCAT results and those just mentioned is that some of the values of $u / d$ ratios calculated with YSCAT data for V-pol are less than unity. This mainly occurs at higher wind speeds and for longer Bragg wavelengths. Both of these factors are consistent with the trends of decreasing $u / d$ with increasing Bragg wavelength and decreasing $u / d$ with increasing wind speed. Nevertheless, the behavior of the YSCAT $u / d$ ratio as a function of polarization, Bragg wavelength, and wind speed is in good agreement with previous results.

\section{E. Discussion}

To summarize, the upper plot of Fig. 10 gives $\gamma$ as a function of Bragg wavelength for all cases previously analyzed. The results for all cases are similar, with the exception of the H-pol upwind case which deviates the most from the mean. The curves imply that $\sigma_{\mathrm{VV}}^{o}$ is more sensitive to wind speed than $\sigma_{\mathrm{HH}}^{\circ}$. An additional conclusion is that smaller Bragg wavelengths (less than $4 \mathrm{~cm}$ ) are much more sensitive to wind speed than are the longer ones. Other experimental results imply that the sensitivity begins to saturate for Bragg wavelengths somewhere less than $2 \mathrm{~cm}$. However, YSCAT data only provides limited samples of $\Lambda$ less than $2 \mathrm{~cm}$ and can not support this conclusion.

The lower plot of Fig. 10 displays the $\gamma_{U} / \gamma_{D}$ and $\gamma_{\mathrm{HH}} / \gamma_{\mathrm{VV}}$ ratios as a function of Bragg wavelength $\Lambda$. The $\gamma_{\mathrm{HH}} / \gamma_{\mathrm{VV}}$ ratios exhibit a decreasing trend with decreasing Bragg wavelength. For the wind speed sensitive wavelengths $(2-4 \mathrm{~cm})$, the $\gamma_{\mathrm{HH}} / \gamma_{\mathrm{VV}}$ ratio is less than unity which suggests that $\sigma_{\mathrm{VV}}^{\circ}$ is 

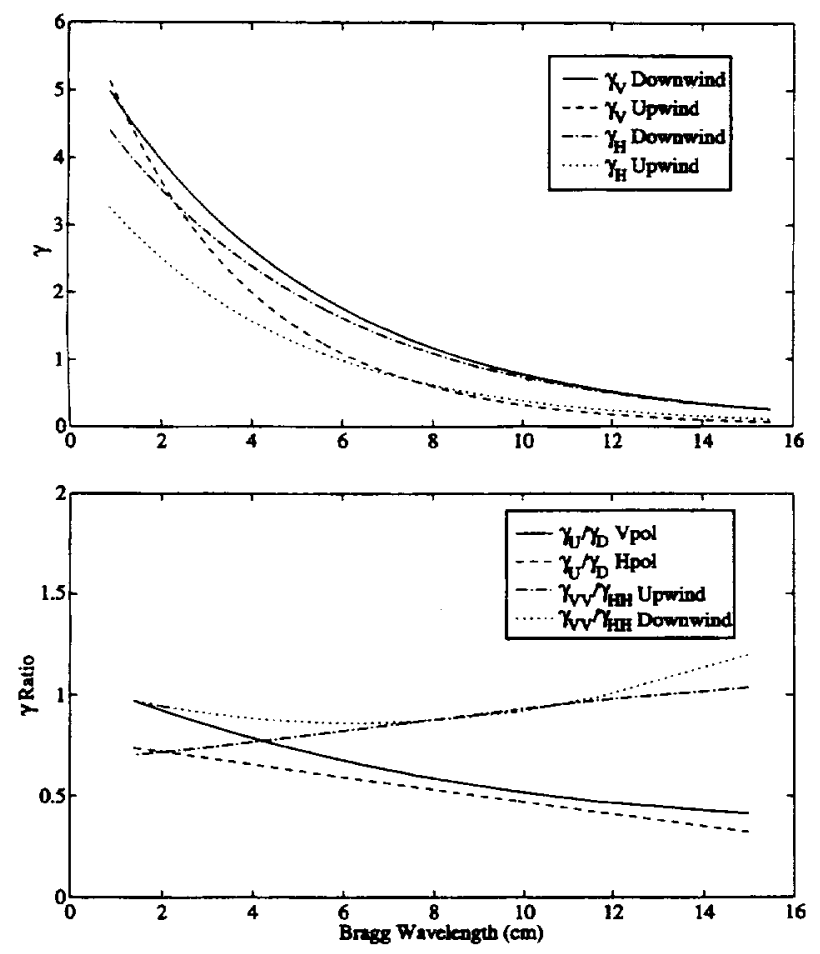

Fig. 10. Top: Wind speed exponents $(\gamma)$ as a function of Bragg wavelength. Bottom: Ratios of $\gamma_{U} / \gamma_{D}$ and $\gamma_{\mathrm{HH}} / \gamma_{\mathrm{VV}}$ as a function of Bragg wavelength.

more sensitive to wind speed. Both the $\gamma_{U} / \gamma_{D}$ ratios increase with decreasing Bragg wavelength. Notice that for $\Lambda$ less than $4 \mathrm{~cm}, \gamma_{U} / \gamma_{D}$ for V-pol approaches unity while $\gamma_{U} / \gamma_{D}$ for $\mathrm{H}$-pol is lower. This result implies that the upwind/downwind ratio of $\sigma^{\circ}$ for $\mathrm{H}$-pol will have a greater change as a function of wind speed.

Fig. 11 presents $u / d$ ratios for $V$-pol (denoted $u / d \mathrm{vv}$ ) and H-pol (denoted $u / d_{\mathrm{HH}}$ ) at four different wind speeds. In both cases two trends are apparent. The first is that the $u / d$ ratio increases with decreasing Bragg wavelength, and the second is that the $u / d$ ratio decreases with increasing wind speed. The first result implies that shorter Bragg wavelengths are more sensitive to relative azimuth (wind direction) than are longer wavelengths. Another important observation is that $u / d_{\mathrm{HH}}$ is higher at all wind speeds than $u / d_{\mathrm{Vv}}$. This suggests that $\sigma_{\mathrm{HH}}^{o}$ is more sensitive to wind direction than $\sigma_{\mathrm{VV}}^{\circ}$.

Assuming that the general trends noted in this study also apply to an oceanic environment, some implications in the remote sensing of winds from spaceborne scatterometers can be identified. Practical reasons such as cost and complexity imply that a spaceborne scatterometer should be a single frequency radar. Moreover, the incidence angle covers moderate Bragg scattering regions. Given this system, what should the operating frequency be? Fig. 12 illustrates graphically which Bragg wavelengths are attainable at moderate incidence angles for C-band, X-band, and Ku-band. In this plot horizontal dotted lines $(\Lambda=2 \mathrm{~cm}, \Lambda=4 \mathrm{~cm})$ have been added to emphasize the region where $\sigma^{\circ}$ is most sensitive to wind speed, while the vertical dotted lines $\left(\theta=30^{\circ}, \theta=50^{\circ}\right)$ highlight the incidence angle range used in this study. The results presented in this paper have shown that $\sigma^{\circ}$ is most sensitive to wind speed and
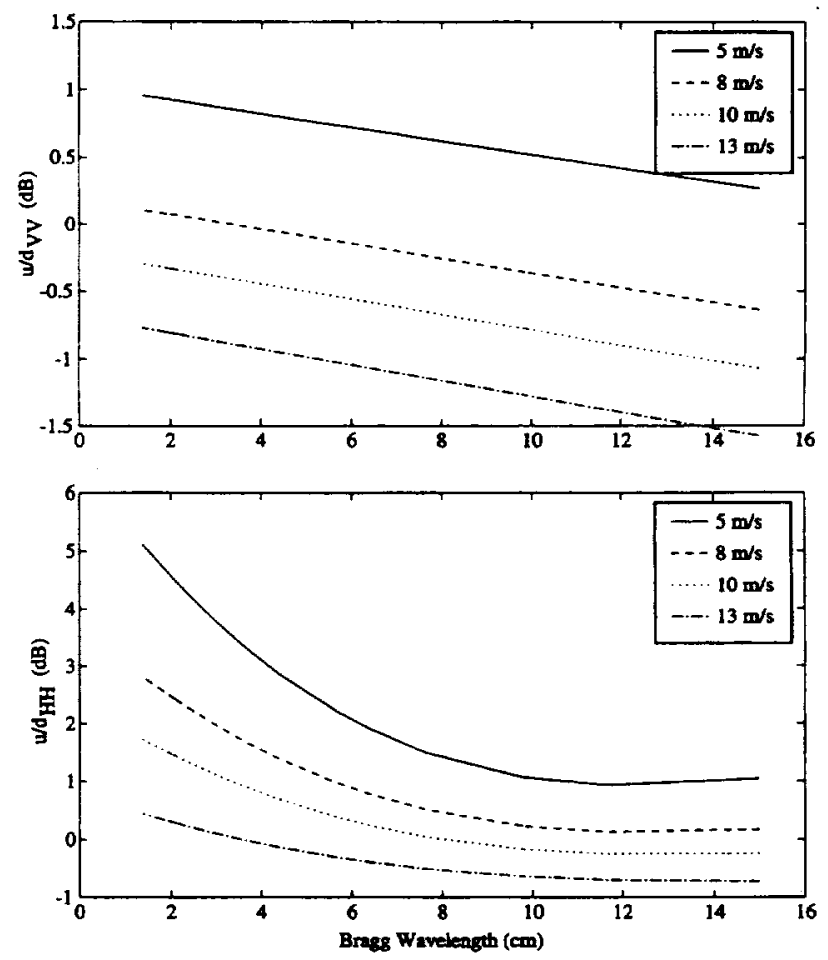

Fig. 11. $\sigma^{\circ}$ upwind/downwind ratio for both polarizations as function of Bragg wavelength for wind speeds of $5,8,10$, and $13 \mathrm{~m} / \mathrm{s}$.

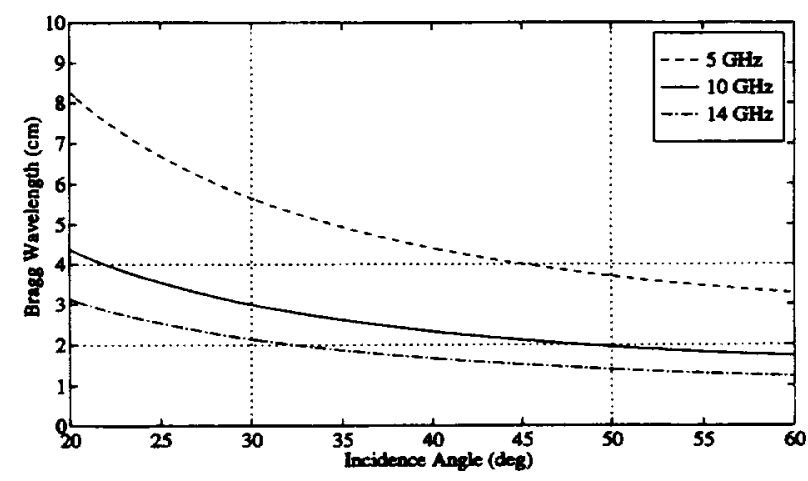

Fig. 12. Bragg wavelengths as a function of both frequency and incidence angle. The dotted lines have been added to emphasize the "optimum" region of Bragg wavelengths and incidence angles. See text.

has a higher $u / d$ ratio for short Bragg wavelengths. Given this, Fig. 12 suggests that either Ku-band or X-band may be better operational frequencies than $\mathrm{C}$-band. From the results of this study, it is difficult to further distinguish between $\mathrm{X}$-band and Ku-band.

Although $\sigma^{\circ}$ at $\mathrm{X}$-band and Ku-band appears to be more sensitive to wind speed and direction than at C-band, the values of $\sigma^{\circ}$ at the higher frequencies also exhibit much higher variability. This variability may be due to other unmodeled parameters such as air-sea temperature difference and long wave fields. In this respect, $\mathrm{C}$-band may be a better operational frequency since the measurements appear to be less sensitive to other environmental parameters. C-band measurements are also less sensitive to atmospheric attenuation due to the presence of liquid water and water vapor. Accounting for envi- 
ronmental parameters such as air-sea temperature differences, atmospheric attenuation, and long wave fields on a global scale may be very difficult in an operational system and would require a better understanding of these effects than is presently available.

Regarding polarization, V-pol appears to be slightly more sensitive to wind speed, but $\mathrm{H}$-pol is much more sensitive to wind direction. In addition, the measurement variability at 10 and $14 \mathrm{GHz}$ is lower for $\mathrm{H}$-pol. The results suggest that $\mathbf{H}$ pol may be better than V-pol in determining both wind speed and direction, while $V$-pol appears superior in measuring wind speed only. It should be noted, however, that because $\mathrm{H}$-pol $\sigma^{o}$ is generally lower than $\mathrm{V}$-pol $\sigma^{\circ}$ and the signal-to-noise ratio of the $\mathrm{H}$-pol measurements will be lower than for $\mathrm{V}$-pol, possibility negating any advantage offered by the improved wind sensitivity of $\mathrm{H}$-pol. We also note that these conclusions are based on only three months of YSCAT 1994 data; however, our data is generally consistent with results published by other researchers.

\section{CONCLUSION}

An ultrawideband scatterometer, YSCAT, has been developed, tested, and deployed on the CCIW WAVES Research Tower. Together with the weather sensor data, YSCAT data is being collected to support a variety of ocean scattering studies. This paper investigates the wind speed and direction sensitivity of the normalized radar cross section $\sigma^{\circ}$ using three months of YSCAT data.

The data set taken from approximately May 6-Aug. 1, 1994, is edited to focus on moderate incidence angles $\left(20-50^{\circ}\right)$, upwind and downwind relative azimuth angles, and wind speeds from $4.5-14 \mathrm{~m} / \mathrm{s}$. Assuming a power law relation between $\sigma^{\circ}$ and wind speed, least squares linear regression is computed to determine the wind speed exponent $(\gamma)$ for each frequency, polarization, incidence angle, and relative azimuth direction.

The wind speed exponent $(\gamma)$ is examined as both a function of incidence angle and Bragg wavelength. The plots of $\gamma$ versus incidence angle $(\theta)$ show that $\gamma$ typically increases with increasing $\theta$ from $20-50^{\circ}$. Most cases display a peak in $\gamma$ at $50^{\circ}$; however, the results at $2 \mathrm{GHz}$ show little apparent dependence on $\theta$. As a function of Bragg wavelength $(\Lambda)$, all results show that $\gamma$ increases with decreasing $\Lambda$. In comparison to previous studies, $\gamma$ is much higher for small $\Lambda$ especially at $V$-pol. This difference may be attributed to differences in the drag coefficient of Lake Ontario and that of the open ocean.

Using the regression fits, the upwind/downwind $(u / d)$ ratio of $\sigma^{\circ}$ is determined as a function of wind speed and Bragg wavelength. The data display two important trends. First, the $u / d$ ratio increases with decreasing $\Lambda$ and second, the $u / d$ ratio decreases with increasing wind speed. These trends are consistent with results published by other researchers.

Using a least squares exponential or polynomial fit, the behavior of $\gamma$ and $u / d$ are compared. Analysis of these comparisons gives the following conclusions.

- V-pol $\sigma^{\circ}$ is slightly more sensitive than H-pol $\sigma^{\circ}$ to wind speed.
- H-pol $\sigma^{\circ}$ is more sensitive than V-pol $\sigma^{\circ}$ to wind direction.

- Bragg wavelengths less than $4 \mathrm{~cm}$ are the most sensitive to wind speed and direction.

\section{ACKNOWLEDGMENT}

The authors thank Dr. M. Donelan and the Canadian Centre for Inland Waters for assisting them with this experiment.

\section{REFERENCES}

[1] A. H. Chaudhry and R. K. Moore, "Tower-based backscatter measurements of the sea," IEEE J. Ocean. Eng., vol. OE-9, no. 5, pp. 309-316, 1984.

[2] R. S. Collyer, "Dependence of the normalized radar cross section of ocean waves on Bragg wavelength-Wind speed and direction sensitivity," Master's thesis, Brigham Young Univ., Provo, UT, 1994.

[3] M. C. Colton, W. J. Plant, W. C. Keller, and G. L. Geernaert, "Towerbased measurements of normalized radar cross section from Lake Ontario: Evidence of wind stress dependence," J. Geophys. Res., vol. 100, no. C5, pp. 8791-8813, 1995.

[4] M. A. Donelan and W. J. Pierson, Jr., "Radar scattering and equilibrium ranges in wind-generated waves with application to scatterometry," $J$. Geophys. Res., vol. 92, pp. 4971-5029, 1987.

[5] M. A. Donelan, J. Hamilton, and W. Hui, "Directional spectra of wind-generated waves," Phil. Trans. Rol. Soc. London, vol. A315, pp. 509-562, 1985.

[6] F. Feindt, V. Wismann, W. Alpers, and W. C. Keller, “Airborne measurements of the ocean radar cross section at $5.3 \mathrm{GHz}$ as a function of wind speed," Radio Sci., vol. 21. pp. 845-856, 1986.

[7] A. T. Jessup, "Detection and characterization of deep water wave breaking using moderate incidence angle microwave backscatter from the sea surface," Ph.D. dissertation, Mass. Inst. Technol., Cambridge, 1990 , Oct. 1995.

[8] W. C. Keller, V. Wismann, and W. Alpers, "Tower-based measurements of the ocean C band radar backscatting cross section," J. Geophys. Res., vol. 94, no. C1, pp. 924-930, Jan. 15, 1989.

[9] W. C. Keller and W. J. Plant. "Cross sections and modulation transfer functions at $\mathrm{L}$ and $\mathrm{Ku}$ bands measured during the tower ocean wave and radar dependence experiment," J. Geophys. Res., vol. 95, no. C9, pp. 16277-16289, Sept. 15, 1990

[10] M. R. Keller, W. C. Keller, and W. J. Plant, "A wave tank study of the dependence of the $\mathrm{X}$-band cross sections on wind speed and water temperature," J. Geophys. Res., vol. 97, no. C4, pp. 5771-5792, 1992.

[11] W. C. Keller and W. J. Plant, "Cross sections and modulation transfer functions at $\mathrm{L}$ and $\mathrm{Ku}$ bands measured during the tower ocean wave and radar dependence experiment," J. Geophys. Res., vol. 95, no. C9, pp. $16277-16289$, Sept. 15, 1990.

[12] H. Masuko, K. Okamoto, M. Shimada, and S. Niwa, "Measurement of microwave backscattering signatures of the ocean surface using X-band and Ka-band airborne scatterometers," J. Geophys. Res., vol. 91, no. C11, pp. 13065-13 083, Nov. 15, 1986.

[13] F. Naderi, M. H. Freilich, and D. G. Long, "Spaceborne radar measurement of wind velocity over the ocean-an overview of the NSCAT scatterometer system," Proc. IEEE, vol. 79, no. 6, pp. 850-866, June 1991.

[14] W. J. Plant, E. A. Terray, R. A. Petitt, and W. C. Keller, "The dependence of microwave backscatter from the sea on illuminated area: correlation times and lengths," J. Geophys. Res., vol. 99, pp. 9705-9723, 1994.

[15] W. J. Plant, "A two-scale model of short wind-generated waves and scatterometry," J. Geophys. Res., vol. 91, pp. 10735-10749, 1986.

[16] R. Reed, D. G. Long, D. V. Amold, and R. S. Collyer, "Initial results from the deployment of an ultra-wide band scatterometer," in International Geoscience and Remote Sensing Symposium, T. I. Stien, Ed. Piscataway, NJ: IEEE Press, pp. 799-801, 1994.

[17] F. T. Ulaby, R. K. Moore, and A. K. Fung, Microwave Remote Sensing. Active and Passive, vol. 2. Norwood, MA: Artech House Inc., 1981.

[18] C. M. H. Unal, P. Snoeij, and P. J. F. Swar, "The polarization-dependent relation between radar backscatter from the ocean surface and surface wind vector at frequencies between 1 and $18 \mathrm{GHz}$," IEEE Trans. Geosci. Remote Sensing, vol. 29, no. 4, pp. 621-626, July 1991.

[19] F. J. Wentz, S. Peteherych, and L. A. Thomas, "A model function for ocean radar cross-sections at $14.6 \mathrm{GHz}$," J. Geophys. Res., vol. 89, pp. 3689-3704, May 20, 1984 
David G. Long (S'80-M'82), for a photograph and biography, see p. 424 of the March 1996 issue of this TRANSACTIONS.

R. Scott Collyer, photograph and biography not available at the time of publication.

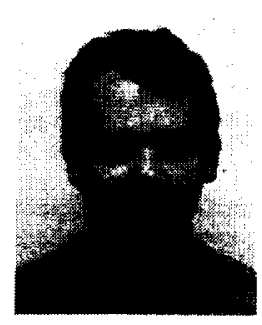

Ryan Reed (S'92-M'93) received the B.S. degree in electrical engineering in 1992 from Brigham Young University, Provo, UT, where he is currently pursuing the Ph.D degree.

He is currently a Research Assistant in the BYU Microwave Earth Remote Sensing Laboratory. His research activities include microwave scattering and radar systems.

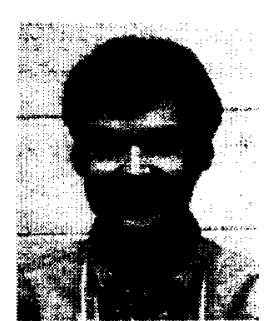

David V. Arnold received the B.S and M.S. degrees from Brigham Young University, Provo, UT, and the Ph.D. degree from the Massachussetts Institute of Technology, Cambridge.

His research interests are in electromagnetic theory and microwave remote sensing. He is currently an Assistant Professor in the Electrical and Computer Engineering Department at Brigham Young. 
